\title{
Probing the hydrophobic cavity of Lipid Transfer Protein from Nicotiana tabacum through Xenon-based NMR Spectroscopy Supporting Information
}

Lionel Dubois, ${ }^{1}$ Pedro Da Silva, ${ }^{2}$ Céline Landon, ${ }^{2}$ J. Gaspard Huber, ${ }^{1}$ Michel

Ponchet, ${ }^{3}$ Françoise Vovelle, ${ }^{2}$ Patrick Berthault, ${ }^{1}$ and Hervé Desvaux ${ }^{1, *}$

${ }^{1}$ Laboratoire Commun de R.M.N., DSM/DRECAM/Service de Chimie Moléculaire, URA CEA/CNRS 331 Claude Fréjacques, C.E.A./Saclay, F-91191 Gif sur Yvette, France

${ }^{2}$ Centre de Biophysique Moléculaire, UPR 4301 CNRS affiliated to Orléans University, Rue Charles Sadron, 45071 Orléans cedex 2, France

${ }^{3}$ UMR Interactions Plantes-Microorganismes et Santé Végétale, INRA, 400 route des Chappes, BP 167, 06903 Sophia Antipolis cedex, France

(Dated: September 27, 2004)

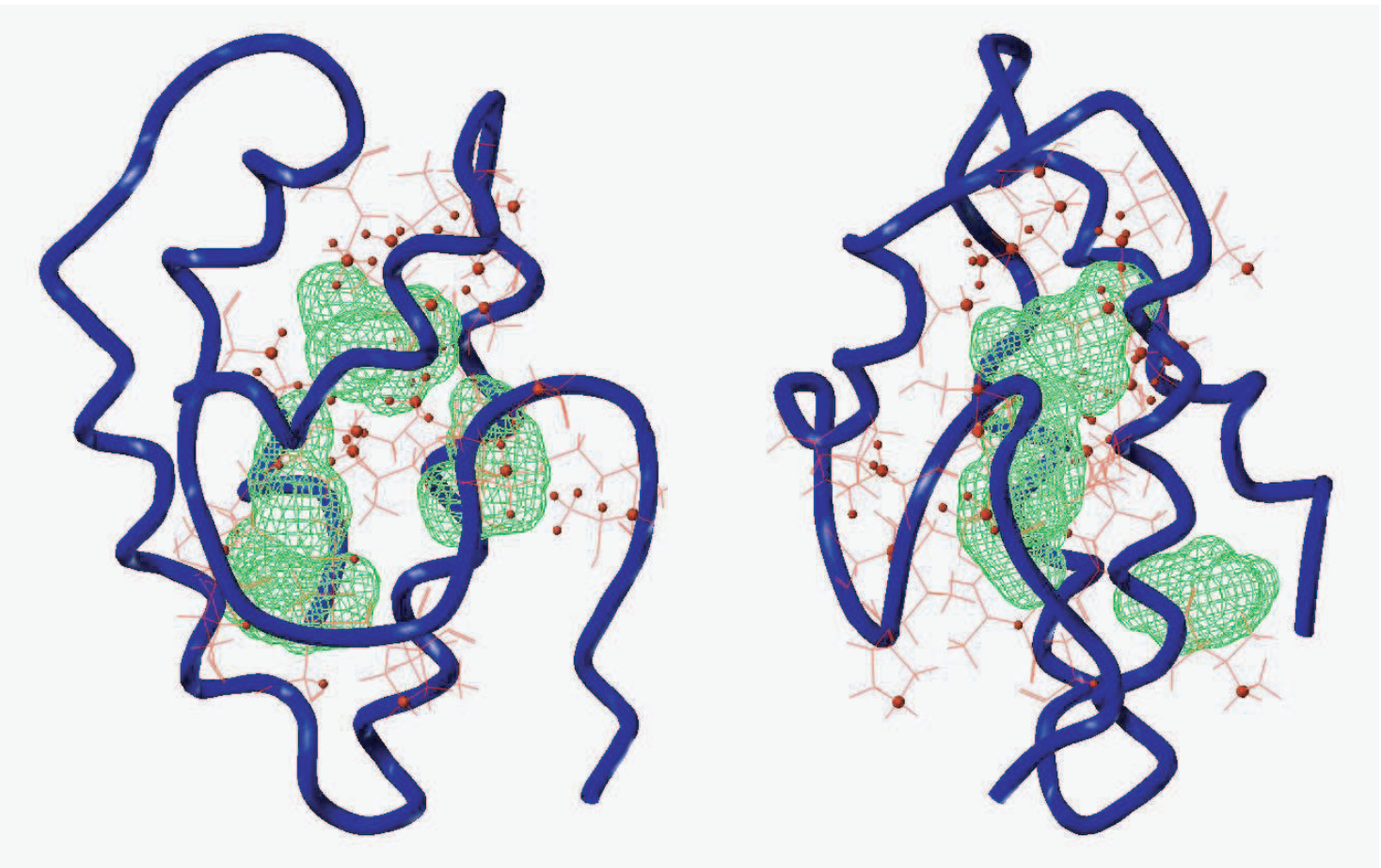

FIG. 1: Structure of tobacco LTP1_1. The ${ }^{1} \mathrm{H}$ and ${ }^{13} \mathrm{C}$ atoms exhibiting a chemical shift variation under xenon pressure are represented by a sphere and the amino-acids they belong in stick mode. The hydrophobic cavity is also represented. 


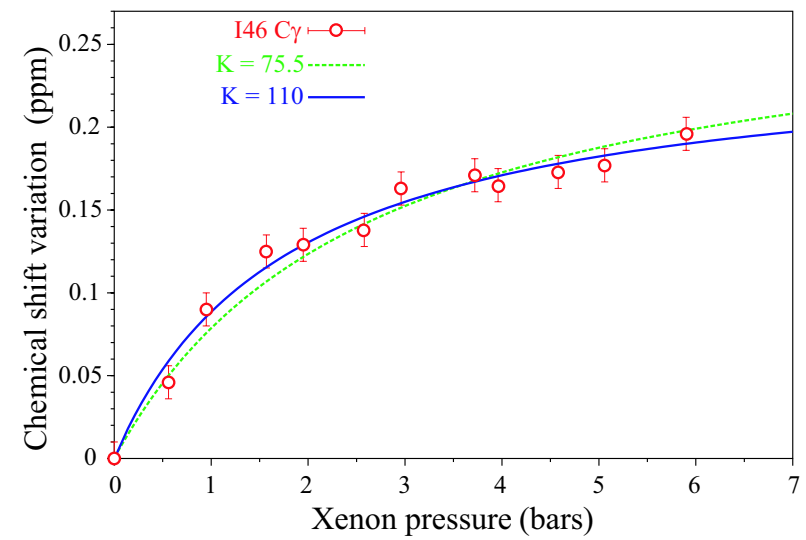

FIG. 2: Experimental variation of the ${ }^{13} \mathrm{C}$ chemical shift of $\mathrm{C}_{\gamma_{1}}$ of $\mathrm{I} 46$ as a function of the xenon pressure. The best fit theoretical curves to the specific model using $K=75.5 \mathrm{M}^{-1}$, (best-fit value for all chemical shift variations, dashed line) and $K=110.0 \mathrm{M}^{-1}$ (best fit value for site $\mathrm{D}$, solid line) are superimposed. 


\begin{tabular}{llll} 
Residue & Atom & $\Delta \delta_{\text {complex }}$ error \\
A12 & $\mathrm{N}^{\mathrm{H}}$ & 0.91 & 0.02 \\
L34 & $\mathrm{N}^{\mathrm{H}}$ & -0.25 & 0.02 \\
S37 & $\mathrm{N}^{\mathrm{H}}$ & 0.26 & 0.02 \\
A38 & $\mathrm{N}^{\mathrm{H}}$ & 0.37 & 0.02 \\
T40 & $\mathrm{N}^{\mathrm{H}}$ & 0.27 & 0.02 \\
S53 & $\mathrm{N}^{\mathrm{H}}$ & 0.34 & 0.02 \\
G56 & $\mathrm{N}^{\mathrm{H}}$ & -0.67 & 0.02 \\
I58 & $\mathrm{N}^{\mathrm{H}}$ & 0.29 & 0.02 \\
A67 & $\mathrm{N}^{\mathrm{H}}$ & -1.34 & 0.02 \\
V75 & $\mathrm{N}^{\mathrm{H}}$ & -0.42 & 0.02 \\
S82 & $\mathrm{N}^{\mathrm{H}}$ & 0.40 & 0.02 \\
S84 & $\mathrm{N}^{\mathrm{H}}$ & -0.44 & 0.02 \\
\hline T3 & $\mathrm{H}^{\mathrm{N}}$ & -0.077 & 0.003 \\
T8 & $\mathrm{H}^{\mathrm{N}}$ & -0.049 & 0.003 \\
N10 & $\mathrm{H}^{\mathrm{N}}$ & 0.094 & 0.003 \\
C14 & $\mathrm{H}^{\mathrm{N}}$ & -0.037 & 0.003 \\
Y17 & $\mathrm{H}^{\mathrm{N}}$ & 0.037 & 0.003 \\
L18 & $\mathrm{H}^{\mathrm{N}}$ & -0.059 & 0.003 \\
V31 & $\mathrm{H}^{\mathrm{N}}$ & -0.096 & 0.003 \\
A33 & $\mathrm{H}^{\mathrm{N}}$ & 0.044 & 0.003 \\
L34 & $\mathrm{H}^{\mathrm{N}}$ & -0.069 & 0.003 \\
A38 & $\mathrm{H}^{\mathrm{N}}$ & 0.086 & 0.003 \\
T40 & $\mathrm{H}^{\mathrm{N}}$ & 0.082 & 0.003 \\
Q45 & $\mathrm{H}^{\mathrm{N}}$ & 0.051 & 0.003 \\
I46 & $\mathrm{H}^{\mathrm{N}}$ & 0.069 & 0.003 \\
L51 & $\mathrm{H}^{\mathrm{N}}$ & 0.050 & 0.003 \\
S53 & $\mathrm{H}^{\mathrm{N}}$ & 0.132 & 0.003 \\
G56 & $\mathrm{H}^{\mathrm{N}}$ & -0.070 & 0.003 \\
I58 & $\mathrm{H}^{\mathrm{N}}$ & 0.083 & 0.003 \\
I61 & $\mathrm{H}^{\mathrm{N}}$ & -0.074 & 0.003 \\
A67 & $\mathrm{H}^{\mathrm{N}}$ & -0.085 & 0.003 \\
G68 & $\mathrm{H}^{\mathrm{N}}$ & 0.045 & 0.003 \\
C73 & $\mathrm{H}^{\mathrm{N}}$ & -0.059 & 0.003 \\
V75 & $\mathrm{H}^{\mathrm{N}}$ & 0.035 & 0.003 \\
Y79 & $\mathrm{H}^{\mathrm{N}}$ & -0.066 & 0.003 \\
K80 & $\mathrm{H}^{\mathrm{N}}$ & 0.101 & 0.003 \\
S82 & $\mathrm{H}^{\mathrm{N}}$ & 0.148 & 0.003 \\
C87 & $\mathrm{H}^{\mathrm{N}}$ & -0.062 & 0.003
\end{tabular}

TABLE I: List of the amide nitrogen and proton experiencing specific chemical shift variation under xenon pressure. The value $\Delta \delta_{\text {complex }}$ is computed for $K=75.5 \mathrm{M}^{-1}$. 


\begin{tabular}{|c|c|c|c|}
\hline Residue & Atom & $\Delta \delta_{\text {complex }}$ & error \\
\hline L11 & $\mathrm{C}_{\beta}$ & -0.206 & 0.006 \\
\hline L11 & $\mathrm{C}_{\delta_{1}}$ & 1.660 & 0.006 \\
\hline L18 & $\mathrm{C}_{\delta_{2}}$ & 1.020 & 0.006 \\
\hline 46 & $\mathrm{C}_{\gamma_{1}}$ & 0.287 & 0.006 \\
\hline 51 & $\mathrm{C}_{\delta_{2}}$ & 0.441 & 0.006 \\
\hline 454 & $\mathrm{C}_{\alpha}$ & 0.155 & 0.006 \\
\hline A54 & $\mathrm{C}_{\beta}$ & 0.369 & 0.006 \\
\hline A55 & $\mathrm{C}_{\alpha}$ & 0.274 & 0.006 \\
\hline I58 & $\mathrm{C}_{\delta_{1}}$ & 0.421 & 0.006 \\
\hline I61 & $\mathrm{C}_{\alpha}$ & -0.318 & 0.006 \\
\hline I61 & $\mathrm{C}_{\gamma_{2}}$ & 0.354 & 0.006 \\
\hline I61 & $\mathrm{C}_{\delta_{1}}$ & 0.824 & 0.006 \\
\hline A66 & $\mathrm{C}_{\beta}$ & 0.448 & 0.006 \\
\hline V75 & $\mathrm{C}_{\beta}$ & -0.153 & 0.006 \\
\hline I81 & $\mathrm{C}_{\gamma_{2}}$ & 1.700 & 0.006 \\
\hline I81 & $\mathrm{C}_{\delta_{1}}$ & 0.437 & 0.006 \\
\hline T85 & $\mathrm{C}_{\alpha}$ & 0.221 & 0.006 \\
\hline L11 & $\mathrm{H}_{\beta_{2}}$ & 0.102 & 0.004 \\
\hline L11 & $\mathrm{H}_{\delta_{1}}$ & 0.037 & 0.004 \\
\hline L11 & $\mathrm{H}_{\delta_{2}}$ & 0.043 & 0.004 \\
\hline L18 & $\mathrm{H}_{\delta_{2}}$ & 0.030 & 0.004 \\
\hline L34 & $\mathrm{H}_{\beta_{2}}$ & -0.038 & 0.004 \\
\hline V35 & $\mathrm{H}_{\alpha}$ & -0.051 & 0.004 \\
\hline A38 & $\mathrm{H}_{\alpha}$ & -0.036 & 0.004 \\
\hline L51 & $\mathrm{H}_{\delta_{2}}$ & 0.034 & 0.004 \\
\hline K52 & $\mathrm{H}_{\alpha}$ & -0.033 & 0.004 \\
\hline A54 & $\mathrm{H}_{\beta}$ & 0.033 & 0.004 \\
\hline A 55 & $\mathrm{H}_{\alpha}$ & -0.045 & 0.004 \\
\hline I58 & $\mathrm{H}_{\delta_{1}}$ & -0.040 & 0.004 \\
\hline I61 & $\mathrm{H}_{\gamma_{1}}$ & 0.051 & 0.004 \\
\hline P70 & $\mathrm{H}_{\alpha}$ & -0.065 & 0.004 \\
\hline I77 & $\mathrm{H}_{\gamma_{2}}$ & -0.037 & 0.004 \\
\hline I 81 & $\mathrm{H}_{\gamma_{2}}$ & 0.035 & 0.004 \\
\hline T85 & $\mathrm{H}_{\beta}$ & -0.038 & 0.004 \\
\hline $\mathrm{T} 85$ & $\mathrm{H}_{\gamma_{2}}$ & -0.046 & 0.004 \\
\hline$\overline{\mathrm{A} 57}$ & $\mathrm{C}_{\beta}$ & 0.450 & 0.006 \\
\hline P78 & $\mathrm{C}_{\gamma}$ & 0.194 & 0.006 \\
\hline K80 & $\mathrm{C}_{\beta}$ & -0.214 & 0.006 \\
\hline S82 & $\mathrm{C}_{\alpha}$ & 0.222 & 0.006 \\
\hline K80 & $\mathrm{H}_{\beta_{2}}$ & 0.029 & 0.004 \\
\hline K80 & $\mathrm{H}_{\gamma_{2}}$ & -0.026 & 0.004 \\
\hline
\end{tabular}

TABLE II: List of the aliphatic carbon and proton experiencing specific chemical shift variation under xenon pressure. The value $\Delta \delta_{\text {complex }}$ is computed for $K=75.5 \mathrm{M}^{-1}$. 


\begin{tabular}{lll} 
Residue & Atoms & Site \\
L11 & $\mathrm{Me}_{\delta_{1}}, \mathrm{Me}_{\delta_{2}}$ & $\mathrm{~A}$ \\
L18 & $\mathrm{Me}_{\delta_{1}}, \mathrm{Me}_{\delta_{2}}$ & $\mathrm{~A}$ \\
L51 & $\mathrm{Me}_{\delta_{2}}$ & $\mathrm{~A}$ \\
A55 & $\mathrm{Me}_{\beta}$ & $\mathrm{A}$ \\
L69 & $\mathrm{Me}_{\delta_{2}}$ & $\mathrm{~A}$ \\
I81 & $\mathrm{Me}_{\gamma_{2}}$ & $\mathrm{~A}$ \\
\hline Y79 & $\mathrm{H}_{\delta_{1}}, \mathrm{H}_{\epsilon_{1}}$ & $\mathrm{~B}$ \\
I81 & $\mathrm{H}_{\alpha}$ & $\mathrm{B}$ \\
T85 & $\mathrm{Me}_{\gamma_{2}}$ & $\mathrm{~B}$ \\
\hline V31 & $\mathrm{Me}_{\gamma_{1}}, \mathrm{Me}_{\gamma_{2}} \mathrm{C}$ \\
L34 & $\mathrm{Me}_{\delta_{1}}, \mathrm{Me}_{\delta_{2}} \mathrm{C}$ \\
L51 & $\mathrm{Me}_{\delta_{1}}$ & $\mathrm{C}$ \\
L69 & $\mathrm{Me}_{\delta_{1}}$ & $\mathrm{C}$ \\
V75 & $\mathrm{Me}_{\gamma_{1}}, \mathrm{Me}_{\gamma_{2}} \mathrm{C}$ \\
I77 & $\mathrm{Me}_{\gamma_{2}}, \mathrm{Me}_{\delta_{1}} \mathrm{C}$ \\
I81 & $\mathrm{Me}_{\delta_{1}}$ & $\mathrm{C}$ \\
\hline V7 & $\mathrm{Me}_{\gamma_{1}}$ & $\mathrm{D}$ \\
I46 & $\mathrm{Me}_{\gamma_{2}}$ & $\mathrm{D}$ \\
A47 & $\mathrm{Me}_{\beta}$ & $\mathrm{D}$
\end{tabular}

TABLE III: List of the protons and methyl groups unambiguously detected in the SPINOE experiments

\begin{tabular}{lll} 
Residue & Atoms & Site \\
L11 & $\mathrm{H}_{\alpha}, \mathrm{Me}_{\beta}, \mathrm{H}_{\gamma}$ & A \\
L15 & $\mathrm{H}_{\beta, \beta^{\prime}}, \mathrm{Me}_{\delta_{1}}, \mathrm{Me}_{\delta_{2}}$ & $\mathrm{~A}$ \\
L18 & $\mathrm{H}_{\beta_{2}}$ & $\mathrm{~A}$ \\
L51 & $\mathrm{Me}_{\beta}$ & $\mathrm{A}$ \\
$\mathrm{A} 54$ & $\mathrm{H}_{\alpha}, \mathrm{Me}_{\beta}$ & $\mathrm{A}$ \\
$\mathrm{I} 58$ & $\mathrm{Me}_{\gamma_{2}}, \mathrm{Me}_{\delta_{1}}$ & $\mathrm{~A}$ \\
$\mathrm{I} 61$ & $\mathrm{Me}_{\gamma_{2}}$ & $\mathrm{~A}$ \\
$\mathrm{~A} 66$ & $\mathrm{H}_{\alpha}, \mathrm{Me}_{\beta}$ & $\mathrm{A}$ \\
$\mathrm{I} 81$ & $\mathrm{H}_{\beta}$ & $\mathrm{A}$ \\
\hline K52 & $\mathrm{H}_{\beta, \beta^{\prime}}, \mathrm{H}_{\gamma, \gamma^{\prime}}$ & $\mathrm{B}$ \\
S82 & $\mathrm{H}_{\beta_{2}}$ & $\mathrm{~B}$ \\
\hline V31 & $\mathrm{H}_{\beta}$ & $\mathrm{C}$ \\
L34 & $\mathrm{Me}_{\beta}, \mathrm{H}_{\gamma}$ & $\mathrm{C}$ \\
V35 & $\mathrm{H}_{\beta}, \mathrm{Me}_{\gamma_{1}}, \mathrm{Me}_{\gamma_{2}}$ & $\mathrm{C}$ \\
L51 & $\mathrm{H}_{\gamma}$ & $\mathrm{C}$ \\
V75 & $\mathrm{H}_{\alpha}$ & $\mathrm{C}$ \\
I77 & $\mathrm{H}_{\beta}, \mathrm{Me}_{\gamma_{1}}$ & $\mathrm{C}$ \\
\hline $\mathrm{I} 2$ & $\mathrm{H}_{\gamma_{1}}, \mathrm{Me}_{\delta_{1}}$ & $\mathrm{D}$ \\
I46 & $\mathrm{Me}_{\delta_{1}}$ & $\mathrm{D}$
\end{tabular}

TABLE IV: Ambiguous interactions detected in the SPINOE experiments 